\title{
Black-White Differences in Housing Type and Sleep Duration as Well as Sleep Difficulties in the United States
}

\author{
Dayna A. Johnson ${ }^{1}$, Roland J. Thorpe Jr. ${ }^{2}$, John A. McGrath ${ }^{3}{ }^{\mathbb{D}}$, W. Braxton Jackson $\mathrm{II}^{3}$ and \\ Chandra L. Jackson 4 ,* \\ 1 Division of Sleep and Circadian Disorders, Brigham and Women's Hospital, Harvard Medical School, \\ Boston, MA 02131, USA; djohnson@research.bwh.harvard.edu \\ 2 Program for Research on Men's Health, Hopkins Center for Health Disparities Solutions, Johns Hopkins \\ Bloomberg School of Public Health, Baltimore, MD 21205, USA; rthorpe@jhu.edu \\ 3 Social \& Scientific Systems, Inc., Research Triangle Park, NC 27709, USA; JMcgrath@s-3.com (J.A.M.); \\ BJackson@s-3.com (W.B.J.) \\ 4 Epidemiology Branch, National Institute of Environmental Health Sciences, National Institutes of Health, \\ Department of Health and Human Services, 111 TW Alexander Drive, Research Triangle Park, NC \\ 27709, USA \\ * Correspondence: Chandra.Jackson@nih.gov; Tel.: +1-984-287-3701
}

Received: 11 December 2017; Accepted: 16 March 2018; Published: 21 March 2018

\begin{abstract}
Housing environments can directly and indirectly affect sleep, and blacks are more likely than whites to live in suboptimal housing conditions, which may independently contribute to sleep disparities. However, few large-scale epidemiological studies consider the potential influence of housing type on sleep health. Using data from the 2004-2015 National Health Interview Survey, we investigated overall and Black-White differences in the association between housing type (house/apartment versus mobile home/trailer) and sleep duration as well as sleep difficulties among 226,208 adults in the U.S. Poisson regression with robust variance was used to estimate sex-specific prevalence ratios (PR) for sleep categories, first comparing houses/apartments to mobile homes/trailers and then blacks to whites within housing types. All models were adjusted for age, educational attainment, income, occupational class, self-reported general health status, and region of residence. Compared to participants living in houses/apartments, the prevalence of short sleep was higher for men ( $\mathrm{PR}=1.05$ (95\% confidence interval $(\mathrm{CI}): 1.02-1.08)$ ) and women ( $\mathrm{PR}=1.07$ (95\% CI: 1.04-1.09)) in mobile homes/trailers. Black men (PR $=1.26$ (95\% CI: 1.21-1.30)) and women $(\mathrm{PR}=1.24(95 \% \mathrm{CI}: 1.20-1.27))$ in a house/apartment were more likely to be short sleepers than their white counterparts. There was generally no significant difference in sleep characteristics (except long sleep) between black and white men in mobile homes/trailers after adjustments, and black men in houses/apartments as well as black women in both housing types were less likely to report sleep difficulties although being more likely to report short sleep. Overall, individuals in mobile homes/trailers, which may represent suboptimal housing, had worse sleep than those in houses / apartments; and racial differences in the quality of houses and apartments are likely to greatly vary in ways that still contribute to sleep disparities. Race-sex group differences in sleep duration among residents in a house/apartment and even a lack of racial difference among individuals living in mobile homes/trailers support the need for more research on residential environments and eventually multi-level interventions designed to reduce sleep disparities.
\end{abstract}

Keywords: sleep duration; sleep difficulties; housing; race; health disparities 


\section{Introduction}

The home environment-independent of neighborhoods-is an important, understudied determinant of health. Social and environmental exposures, such as safety and social cohesion, crowding, indoor air quality, inopportune light and noise, as well as other ambient conditions [1,2], which have been shown to disturb sleep [3-6], may vary by housing type, such as houses, apartments, and mobile homes. Suboptimal housing is also associated with poor health outcomes (e.g., type 2 diabetes; cardiovascular disease) that are often recognized as a consequence of or exacerbated by poor sleep [1,2,7-9]. Therefore, it is important to identify and understand the potential variation in sleep by housing type. Research on housing and sleep could help prioritize settings and approaches that may improve sleep and reduce the burden of subsequent poor health outcomes that often disproportionately affect racial minorities and other under-resourced populations.

Several epidemiologic studies have found that housing structure impacts sleep [10,11]. For instance, noisy home environments [12-14], household crowding [15,16], and suboptimal ambient temperature [17] were independently associated with insufficient sleep. These different housing characteristics likely vary by housing type and may provide important insights into how physical and social environments influence sleep duration and sleep difficulties. For instance, mobile home/trailer residents may have greater exposure to and less control over inopportune light, temperature, or noise compared to those residing in a house or apartment. Additionally, housing type may represent another marker of socioeconomic status, based on differences in housing structure integrity/quality, size, and aesthetics, which might be informed by the social and environmental conditions in which one dwells. Therefore, housing type may provide important insight regarding those most-at-risk for sleep problems. Also, investigating the role of housing type in relation to sleep patterns may provide a more comprehensive understanding of the impact of contextual factors on sleep.

Prior research regarding the home environment and its impact on health has been mainly conducted among non-Hispanic white adults or children [12-14,18], which limits generalizability to adults and racial/ethnic minorities who are disproportionately affected by poor sleep $[19,20]$. For example, blacks have been consistently shown to have a shorter sleep duration and poorer sleep quality than whites $[19,21]$. Additionally, blacks are particularly more likely than whites to reside in disadvantaged neighborhoods and have adverse housing conditions [22], which are known to contribute to insufficient sleep [23,24]. Therefore, it is plausible that sleep disparities are influenced by a distressed environment, such as suboptimal housing conditions (e.g., substandard structures, overcrowding) that varies by race. Based on prior evidence, which demonstrated that blacks generally have worse sleep than whites, a similar pattern is expected within housing types. Also, the racial disparity in sleep may be exacerbated in more optimal housing environments. Jackson and colleagues previously reported that a larger sleep disparity existed between blacks and whites of higher socioeconomic status than between those of lower socioeconomic status; thus, it is plausible that the racial sleep disparity may be greater in more optimal than suboptimal housing environments [20]. However, racial variations in sleep duration within different housing types in the United States (U.S.) are currently unknown.

Furthermore, racial disparities in sleep by housing type likely exist as a result of differential access to resources due to historical as well as contemporary discriminatory policies/practices [25]. To further understand disparities in sleep, it is important to investigate how sleep duration and sleep difficulties potentially vary by race within housing type. To address this important gap in the literature, we investigated racial differences in the association between housing type (specifically, house/apartment and mobile home/trailer, which is considered a particularly adverse environment) and self-reported sleep duration as well as sleep difficulties using a nationally representative sample of the U.S. We hypothesized that men and women living in a mobile home/trailer would have shorter $(<7 \mathrm{~h})$ and longer $(\geq 9 \mathrm{~h})$ sleep duration and report more sleep difficulties compared to those in a house/apartment; and black men and women would have shorter and longer sleep duration and report more sleep difficulties relative to white men and women in any housing type. The racial disparity 
was also hypothesized to be smaller among those in mobile homes/trailers. Our hypotheses included associations of both short and long sleep duration based on substantial evidence that has demonstrated a U-shaped relationship between sleep duration and adverse cardiovascular outcomes [26].

\section{Methods}

\subsection{National Health Interview Survey}

We analyzed data from the National Health Interview Survey (NHIS), which is a series of cross-sectional, nationally representative surveys that use a three-stage stratified cluster probability sampling design to conduct in-person interviews in the households of non-institutionalized U.S. civilians. A detailed description of NHIS procedures has been published elsewhere [27]. Briefly, a probability sample of households were interviewed by trained interviewers from the U.S. Census Bureau to obtain information about health and sociodemographic characteristics of the sampled household on a continual weekly basis. Data were collected using computer-assisted personal interviewing. A randomly selected adult and child (not included in this analysis) provided more specific health-related information. The final response rate for sample adults was $80.0 \%$ (range: 74.2-83.7\%) for 2004-2015 surveys. The NHIS received written informed consent from each study participant.

\subsection{Study Population}

Study participants self-identified as Non-Hispanic white and Non-Hispanic black (hereafter, white and black) adults who were at least 18 years old. Participants were excluded if they were born outside the U.S. or had missing data on sleep measures or housing type. We excluded non-U.S. born participants because evidence suggests that sleep patterns among U.S. immigrants differ from those among individuals born in the U.S. [28]. Our final sample comprised 226,208 adults.

\subsection{Measures}

\subsubsection{Sleep Duration and Sleep Difficulties}

Sampled adults reported how many hours they sleep, on average, in a 24-h period. By instruction, interviewers reported sleep hours in whole numbers while rounding values of $30 \mathrm{~min}$ or more up to the nearest hour and rounding values less than $30 \mathrm{~min}$ down to the nearest hour. Short sleep duration was defined as a usual sleep duration of less than $7 \mathrm{~h}$; the recommended amount of sleep was defined as 7-8 h; and long sleep was defined as equal or more than $9 \mathrm{~h}$. Seven-to-eight hours of sleep was used as the reference because it is associated with the lowest levels of morbidity and mortality [29]. These measures of sleep duration were available for 2004-2015. Additionally, several measures of sleep difficulties were assessed using questions from NHIS 2013-2015 on "trouble falling asleep", "trouble staying asleep", "waking up most days feeling rested", and "took sleep medication one or more times" (all of these in the previous week).

\subsubsection{Housing Type}

Housing type was assessed with twelve options as living quarters: "House, apartment, flat, condo"; "non-transient hotel, motel"; "permanent in transient hotel, motel"; "rooming house"; "Mobile home/trailer w/no permanent rooms added"; "Mobile home/trailer w/1+ permanent rooms added"; "not specified"; "Quarters not housing unit in room or board house"; "Unit not permanent-transient hotel, motel"; "Unoccupied site for mobile home/trailer/tent"; "Student quarters in college dormitory"; and "Group quarter unit not specified above". The above options were then categorized into four types of housing: "house, apartment/condo", "mobile home/trailer", "dormitory", and "hotel". This analysis focused on the "house, apartment/condo" $(N=213,874)$ and "mobile home/trailer" 
$(N=12,334)$ housing types because the dormitory setting included one demographic of young adult college students and the sample size for hotel was too small for robust estimation.

\subsubsection{Race/Ethnicity}

Participants were asked, "What race or races do you consider yourself to be?" They then selected $\geq 1$ of the following categories: American Indian/Alaskan native, Asian, black/African American, white, or multiple races. National origin or ancestry refers to the national or cultural group from which the person is descended, and ethnicity is classified as Hispanic or Non-Hispanic. This study focuses on blacks and whites because the largest disparity is often observed between these groups and the underlying social as well as biological (not necessarily genetic) mechanisms leading to differences in sleep duration and subsequent health outcomes are likely to vary by race/ethnicity [25,30]. Whites were used as the comparison group for greater statistical stability because this group has the largest sample size.

\subsubsection{Demographic and Clinical Characteristics}

Socioeconomic status. Socioeconomic status (SES) was based on education, income, and occupational class. Educational attainment was categorized as less than high school (no high school diploma), high school (high school or general equivalency diploma), some college (any education beyond high school), and college graduate and beyond. Annual household income was dichotomized as below $\$ 35,000$ versus $\$ 35,000$ or above. Adults who were working at a paying or nonpaying job during the week prior to the survey, those who had a job or business but were not at work during the prior week, and those who had ever worked were asked about their occupations, which were categorized on the basis of the Standard Occupational Classification System (http://www.bls.gov/soc/). We combined occupations into categories of professional/management, support services, or laborers based on type of work. Class of work/occupation (based on current, longest held, or most recently held job or work situation) was classified as either (1) an employee of a private company, business, or individual for wages, salary, or commission; (2) a federal, state, or local government employee; (3) self-employed in own business, professional practice, or farm; or (4) working without pay in a family-owned business or farm. Furthermore, marital status was categorized as married/living with a partner, divorced/separated/widowed, or never married.

Region of residence. Regions of the country were categorized as South, Midwest, Northeast, or West.

Clinical characteristics. Self-reported height and weight were used to calculate body mass index (BMI). Obesity was defined as a BMI of $\geq 30 \mathrm{~kg} / \mathrm{m}^{2}$, overweight as $25.0-29.9 \mathrm{~kg} / \mathrm{m}^{2}$, normal weight as $18.5-24.9 \mathrm{~kg} / \mathrm{m}^{2}$, and underweight as less than $18.5 \mathrm{~kg} / \mathrm{m}^{2}$ [31]. Self-reported general health status was categorized as "excellent/very good", "good", or "fair/poor".

\subsubsection{Statistical Analysis}

We pooled 12 survey years (2004-2015) of NHIS data merged by the Integrated Health Interview Series. For all analyses, we used a stratification variable and sampling weights that account for the unequal probabilities of selection resulting from the sample design, nonresponse, and oversampling of certain subgroups. Standard errors or variance estimates were calculated by using Taylor series linearization [32]. Stata, version 14, software (StataCorp. LP, College Station, TX, USA) was used for all analyses.

Categorical variables are presented using raw (unweighted) frequencies accompanied by the weighted percentages; additionally, all percentages were standardized to the age structure of the 2010 Census (with three age groups: 18-49, 50-64, and 65+). We used Rao Scott second-order corrected Pearson statistics that take survey weights into account to test for differences in pre-specified sociodemographic, clinical, and behavioral characteristics of interest between whites and blacks, as well as by categories of sleep outcomes. 
We calculated prevalence ratios (PRs) comparing housing types as well as blacks and whites on sleep duration and sleep difficulties as a function of housing type; we used Poisson regression with a robust variance estimator and included sampling weights [33]. Pre-specified socioeconomic, demographic, and clinical characteristics were entered into the model as a group, and white participants were used as the reference for the black-white comparisons. We adjusted for age in three categories (18-49 years, 50-64 years, or $\geq 65$ years) for socioeconomic and demographic factors (income, marital status, educational attainment, and region of residence) and for self-reported health status. Because depression was only available for 2013-2015, it was not included as a potential confounder in regression models. In a secondary data analyses, we additionally adjusted for obesity, type 2 diabetes, and hypertension.

Among men and women separately, we estimated sleep outcomes comparing blacks and whites within housing types.

\section{Results}

Sociodemographic, health behavior, clinical, and healthcare access/utilization characteristics of men and women are shown by housing type and race in Table 1. Of the 226,208 black and white participants, $55 \%$ were female, and $6 \%$ of whites and $4 \%$ of blacks lived in a mobile home/trailer. Blacks were generally younger than whites across housing type, and were more likely to live in poverty even among individuals in mobile homes/trailers.

\subsection{Sociodemographic and Sleep Characteristics by Race and Sex for Houses/Apartments}

Among men who lived in a house/apartment, black men were more likely than white men to have a less than high school education (17\% versus $8 \%$ ), an annual household income less than $\$ 35,000$ ( $45 \%$ versus $24 \%$ ), an occupational status of laborer ( $63 \%$ versus $44 \%$ ), reside in the South $(59 \%$ versus $33 \%$ ), and have fair/poor self-reported general health status ( $21 \%$ versus $11 \%)$. Black men were also more likely than white men to be short ( $36 \%$ versus $27 \%$ ) and long (11\% versus $8 \%$ ) sleepers as well as less likely to obtain the recommended amount of sleep ( $54 \%$ versus $65 \%$ ).

Black women living in a house or apartment were more likely than their white female counterparts to have a less than high school education ( $16 \%$ versus $7 \%$ ), an annual household income less than $\$ 35,000$ ( $56 \%$ versus $30 \%$ ), an occupational status of laborer (29\% versus $16 \%)$, reside in the South $(59 \%$ versus $34 \%)$, and a fair / poor general health status (23\% versus $12 \%)$. Black women were also more likely than white women to be short (37\% versus $27 \%)$ and long (11\% versus $9 \%$ ) sleepers as well as less likely to obtain the recommended amount of sleep (52\% versus $64 \%$ ). 
Table 1. Sociodemographic, Health Behavior, Clinical, and Healthcare Access/Utilization Characteristics among U.S. Adults by Housing Type Stratified by Gender, National Health Interview Survey, 2004-2015 $(N=226,208)$.

\begin{tabular}{|c|c|c|c|c|c|c|c|c|c|c|c|c|c|c|c|c|}
\hline \multirow{3}{*}{ Characteristic $^{a}$} & \multicolumn{8}{|c|}{ Men $(N=102,108)$} & \multicolumn{8}{|c|}{ Women $(N=124,100)$} \\
\hline & \multicolumn{4}{|c|}{ House/Apartment } & \multicolumn{4}{|c|}{ Mobile Home } & \multicolumn{4}{|c|}{ House/Apartment } & \multicolumn{4}{|c|}{ Mobile Home } \\
\hline & White & $\%$ & Black & $\%$ & White & $\%$ & Black & $\%$ & White & $\%$ & Black & $\%$ & White & $\%$ & Black & $\%$ \\
\hline Sample size & 80,857 & 94 & 15,410 & 96 & 5083 & 6 & 758 & 4 & 93,812 & 94 & 23,795 & 96 & 5554 & 6 & 939 & 4 \\
\hline Age, year, mean $\pm \mathrm{SE}$ & 49.7 & 6 & 47.7 & 14 & 50.5 & 24 & 49.2 & 58 & 51.5 & 6 & 47.4 & 12 & 51.3 & 25 & 46.3 & 54 \\
\hline \multicolumn{17}{|l|}{ Educational attainment } \\
\hline$<$ High school & 6153 & 8 & 2479 & 17 & 1141 & 24 & 245 & 34 & 6900 & 7 & 3821 & 16 & 1226 & 23 & 221 & 29 \\
\hline High school graduate & 22,678 & 29 & 5671 & 37 & 2336 & 46 & 351 & 44 & 26,135 & 29 & 7585 & 32 & 2336 & 44 & 401 & 43 \\
\hline Some college & $\begin{array}{l}24,568 \\
24,568\end{array}$ & 29 & 4645 & 29 & $\begin{array}{l}2030 \\
1279\end{array}$ & $\begin{array}{l}40 \\
24\end{array}$ & $\begin{array}{l}531 \\
138\end{array}$ & $\begin{array}{l}44 \\
20\end{array}$ & $\begin{array}{l}20,1103 \\
30,726\end{array}$ & 33 & $\begin{array}{l}7503 \\
8000\end{array}$ & 33 & $\begin{array}{l}2350 \\
1586\end{array}$ & $\begin{array}{l}44 \\
27\end{array}$ & 268 & 24 \\
\hline$\geq$ College & 27,230 & 34 & 2525 & 16 & 298 & 6 & 17 & 3 & 29,792 & 31 & 4253 & 18 & 386 & 6 & 44 & 4 \\
\hline \multicolumn{17}{|l|}{ Marital status } \\
\hline Married & 42,684 & 64 & 5120 & 46 & 2031 & 51 & 262 & 46 & 44,128 & 58 & 4662 & 29 & 2087 & 48 & 229 & 33 \\
\hline Divorced/separated/widowed & 17,485 & 16 & 4443 & 24 & 1899 & 30 & 271 & 30 & 32,324 & 26 & 9083 & 38 & 2645 & 39 & 367 & 38 \\
\hline Never married & 20,521 & 20 & 5795 & 30 & 1136 & 19 & 224 & 24 & 17,103 & 16 & 9928 & 33 & 800 & 13 & 338 & 28 \\
\hline Unemployed & 26,542 & 34 & 6595 & 46 & 2365 & 48 & 372 & 53 & 41,459 & 44 & 10,987 & 49 & 3119 & 57 & 442 & 54 \\
\hline Annual Household income $(<\$ 35,000$ per & 22,101 & 24 & 2943 & 45 & 7324 & 57 & 510 & 68 & 31,831 & 30 & 13,997 & 56 & 3559 & 62 & 687 & 74 \\
\hline $\begin{array}{l}\text { Living in poverty } \\
\text { year }\end{array}$ & 5874 & 6 & 2748 & 17 & 880 & 18 & 211 & 30 & 8839 & 8 & 6572 & 26 & 1267 & 22 & 354 & 38 \\
\hline \multicolumn{17}{|l|}{ Occupation } \\
\hline Professional/management & 21,327 & 28 & 1650 & 11 & 479 & 10 & 26 & 4 & 15,011 & 17 & 2392 & 12 & 376 & 7 & 37 & 4 \\
\hline Support Services & 21,580 & 28 & 3775 & 26 & 719 & 16 & 80 & 11 & 58,258 & 67 & 12,978 & 59 & 2898 & 55 & 374 & 42 \\
\hline Laborers & 34,379 & 44 & 8664 & 63 & 3612 & 75 & 592 & 84 & 14,824 & 16 & 6061 & 29 & 1848 & 38 & 420 & 54 \\
\hline Home ownership & 57,886 & $\begin{array}{l}44 \\
79\end{array}$ & $\begin{array}{l}0004 \\
7345\end{array}$ & 58 & 3824 & 79 & 516 & $\begin{array}{l}04 \\
73\end{array}$ & $\begin{array}{l}14,024 \\
67,037\end{array}$ & 78 & $\begin{array}{l}00011 \\
10,070\end{array}$ & 54 & $\begin{array}{l}1040 \\
4185\end{array}$ & $\begin{array}{l}50 \\
79\end{array}$ & 658 & 73 \\
\hline Govt. Assistance & 4398 & 5 & 2752 & 17 & 843 & 16 & 184 & 24 & 7890 & 7 & 7364 & 27 & 1337 & 23 & 359 & 34 \\
\hline \multicolumn{17}{|l|}{ Region of residence } \\
\hline Northeast & 14,215 & 19 & 1813 & 13 & 485 & 10 & 4 & 1 & 16,778 & 19 & 3210 & 14 & 515 & 9 & 4 & 1 \\
\hline Midwest & 23,172 & 29 & 2927 & 19 & 948 & 17 & 16 & 2 & 26,847 & 29 & 4572 & 19 & 962 & 17 & 13 & 1 \\
\hline South & 25,748 & 33 & 9016 & 59 & 2586 & 55 & 717 & 95 & 30,732 & 34 & 14,015 & 59 & 2919 & 57 & 903 & 96 \\
\hline West & 17,722 & 19 & 1654 & 9 & 1064 & 18 & 21 & 2 & 19,455 & 18 & 1998 & 8 & 1158 & 17 & 19 & 2 \\
\hline \multicolumn{17}{|l|}{ Smoking status } \\
\hline $\begin{array}{l}\text { Mmokng status } \\
\text { Never }\end{array}$ & 38,960 & 48 & 8077 & 51 & 1500 & 29 & 333 & 44 & 54,250 & 58 & 15,876 & 66 & 2252 & 41 & 657 & 66 \\
\hline Former & 24,456 & 32 & 3164 & 24 & 1457 & 30 & 145 & 21 & 22,232 & 24 & 3326 & 16 & 1173 & 22 & 100 & 15 \\
\hline Current & 17,300 & 20 & 4123 & 25 & 2115 & 41 & 277 & 35 & 17,220 & 18 & 4553 & 18 & 2116 & 37 & 177 & 18 \\
\hline \multicolumn{17}{|l|}{ Alcohol consumption } \\
\hline Never & 8473 & 11 & 2978 & 20 & 587 & 12 & 150 & 20 & 17,776 & 19 & 8173 & 36 & 1428 & 27 & 413 & 46 \\
\hline Current & 58,880 & 72 & 9251 & 58 & 3083 & 60 & 415 & 52 & 60,944 & 65 & 11,379 & 45 & 2704 & 48 & 323 & 29 \\
\hline Former & $\begin{array}{l}0,000 \\
12,609\end{array}$ & 16 & 2928 & 22 & 1338 & 28 & 175 & 28 & $\begin{array}{l}0,0,44 \\
14,357\end{array}$ & 15 & 3981 & 19 & 1359 & $\begin{array}{l}40 \\
26\end{array}$ & 189 & 25 \\
\hline $5+$ drinks on at least 2 days $b$ & 21,955 & 33 & 2619 & 26 & 1123 & 34 & 157 & 36 & 11,960 & 16 & 1468 & 11 & 548 & 18 & 46 & 12 \\
\hline Heavy drinking ${ }^{c}$ c & 17,135 & 24 & 1999 & 16 & 795 & 17 & 112 & 18 & 17,600 & 23 & 2128 & 13 & 500 & 12 & 59 & 10 \\
\hline \multicolumn{17}{|l|}{ Leisure-time physical activity } \\
\hline Never/unable & 22,934 & 29 & 5993 & 41 & 2468 & 50 & 409 & 56 & 29,485 & 31 & 11,264 & 49 & 2773 & 51 & 565 & 62 \\
\hline Low & 27,643 & 35 & 4665 & 30 & 1199 & 23 & 181 & 24 & 31,009 & 34 & 6718 & 28 & 1339 & 24 & 201 & 19 \\
\hline High & 29,926 & 36 & 4677 & 29 & 1388 & 27 & 164 & 21 & 33,050 & 35 & 5746 & 23 & 1424 & 25 & 171 & 20 \\
\hline Sad (past 30 days) ( $\geq$ mostly) & 1731 & 2 & 542 & 3 & 255 & 5 & 43 & 6 & 2816 & 3 & 1193 & 4 & 438 & 8 & 59 & 6 \\
\hline Felt depressed (ofte & 1436 & 7 & 274 & 7 & 150 & 15 & 16 & 8 & 2223 & 10 & 490 & 9 & 266 & 22 & 28 & 13 \\
\hline Felt depressed (a lot) & 723 & 12 & 148 & 16 & 75 & 18 & 7 & 16 & 1375 & 15 & 345 & 19 & 164 & 27 & 21 & 24 \\
\hline
\end{tabular}


Table 1. Cont.

\begin{tabular}{|c|c|c|c|c|c|c|c|c|c|c|c|c|c|c|c|c|}
\hline \multirow{3}{*}{ Characteristic a $^{\text {a }}$} & \multicolumn{8}{|c|}{ Men $(N=102,108)$} & \multicolumn{8}{|c|}{ Women $(N=124,100)$} \\
\hline & \multicolumn{4}{|c|}{ House/Apartment } & \multicolumn{4}{|c|}{ Mobile Home } & \multicolumn{4}{|c|}{ House/Apartment } & \multicolumn{4}{|c|}{ Mobile Home } \\
\hline & White & $\%$ & Black & $\%$ & White & $\%$ & Black & $\%$ & White & $\%$ & Black & $\%$ & White & $\%$ & Black & $\%$ \\
\hline \multicolumn{17}{|l|}{ Health outcomes } \\
\hline Overweight prevalence ${ }^{\mathrm{d}}$ & 56,684 & 72 & 10,966 & 72 & 3524 & 71 & 539 & 72 & 49,592 & 53 & 17,271 & 74 & 3620 & 66 & 729 & 76 \\
\hline Obesity prevalence ${ }^{\mathrm{e}}$ & 20,902 & 27 & 4828 & 31 & 1566 & 32 & 251 & 33 & 22,634 & 24 & 9794 & 42 & 1963 & 36 & 460 & 48 \\
\hline Hypertension & 26,146 & 35 & 6180 & 44 & 1999 & 42 & 343 & 49 & 29,149 & 31 & 10,337 & 50 & 2243 & 42 & 471 & 59 \\
\hline Diabetes & 6947 & 10 & 2071 & 16 & 642 & 14 & 120 & 18 & 7161 & 8 & 3094 & 16 & 700 & 14 & 175 & 24 \\
\hline Cancer & 8356 & 11 & 863 & 7 & 539 & 11 & 40 & 8 & 11,595 & 12 & 1252 & 6 & 812 & 14 & 49 & 7 \\
\hline Heart disease & 11,026 & 15 & 1480 & 11 & 864 & 19 & 110 & 16 & 11,198 & 12 & 2447 & 12 & 882 & 16 & 94 & 12 \\
\hline Stroke & 2342 & 3 & 695 & 5 & 271 & 6 & 42 & 6 & 3007 & 3 & 1070 & 5 & 295 & 5 & 62 & 11 \\
\hline Functional limitation & 26,673 & 34 & 4940 & 35 & 2417 & 49 & 311 & 45 & 39,870 & 42 & 9942 & 46 & 3254 & 60 & 405 & 50 \\
\hline Any Injury (3 months) & 2181 & 81 & 366 & 87 & 182 & 93 & 21 & 88 & 2923 & 87 & 608 & 91 & 236 & 96 & 21 & 89 \\
\hline \multicolumn{17}{|l|}{ Healthcare access and utilization } \\
\hline No health insurance & 9041 & 10 & 3082 & 18 & 1302 & 24 & 224 & 26 & 8041 & 8 & 3772 & 14 & 1162 & 21 & 227 & 22 \\
\hline Medicaid & 2980 & 3 & 1836 & 11 & 539 & 10 & 126 & 18 & 6095 & 5 & 5397 & 19 & 987 & 16 & 233 & 22 \\
\hline Usual place of care & 68,121 & 87 & 12,536 & 84 & 3928 & 79 & 607 & 84 & 86,085 & 93 & 21,518 & 92 & 4806 & 86 & 839 & 90 \\
\hline \# ER visits in past year $\geq 2$ & 4395 & 5 & 1554 & 10 & 502 & 10 & 105 & 15 & 6893 & 7 & 3357 & 13 & 808 & 15 & 159 & 17 \\
\hline Delayed healthcare due to cost & 7951 & 8 & 1799 & 9 & 860 & 16 & 111 & 13 & 10,852 & 11 & 3240 & 12 & 1173 & 21 & 134 & 14 \\
\hline HIV testing (ever, yes) & 24,829 & 29 & 8127 & 49 & 1603 & 31 & 304 & 40 & 31,233 & 31 & 13,065 & 49 & 2079 & 36 & 499 & 47 \\
\hline \multicolumn{17}{|l|}{ Health status } \\
\hline Excellent/very good & 51,854 & 63 & 7695 & 47 & 2192 & 41 & 288 & 37 & 59,262 & 63 & 11,161 & 43 & 2300 & 40 & 358 & 34 \\
\hline Good & 19,975 & 25 & 4648 & 32 & 1594 & 32 & 218 & 28 & 23,501 & 25 & 7499 & 33 & 1799 & 33 & 302 & 32 \\
\hline Fair/poor & 8990 & 11 & 3064 & 21 & 1296 & 27 & 251 & 35 & 11,007 & 12 & 5129 & 23 & 1453 & 28 & 278 & 34 \\
\hline \multicolumn{17}{|l|}{ Sleep duration } \\
\hline & 22,845 & 27 & 5678 & 36 & 1842 & 34 & 230 & 29 & 25,889 & 27 & 8759 & 37 & 2055 & 37 & 294 & 31 \\
\hline$\geq 7-<9 \mathrm{~h}$ & 51,640 & 65 & 8241 & 54 & 2697 & 54 & 392 & 52 & 59,285 & 64 & 12,594 & 52 & 2841 & 51 & 508 & 53 \\
\hline$\geq 9 \mathrm{~h}$ & 6372 & 8 & 1491 & 11 & 544 & 12 & 136 & 19 & 8638 & 9 & 2442 & 11 & 658 & 12 & 137 & 16 \\
\hline Trouble falling asleep & 7518 & 30 & 1213 & 28 & 532 & 37 & 52 & 26 & 11,409 & 41 & 2375 & 37 & 730 & 50 & 93 & 37 \\
\hline Trouble staying asleep & 9008 & 38 & 1340 & 32 & 612 & 42 & 53 & 28 & 12,798 & 47 & 2450 & 39 & 784 & 52 & 86 & 33 \\
\hline Most days woke up feeling rested & 16,195 & 68 & 2882 & 69 & 856 & 60 & 145 & 66 & 16,761 & 62 & 3814 & 61 & 745 & 45 & 163 & 70 \\
\hline Times took sleep medication last week $\geq 1$ & 3038 & 12 & 424 & 11 & 232 & 17 & 20 & 8 & 5325 & 19 & 909 & 14 & 361 & 26 & 31 & 10 \\
\hline
\end{tabular}

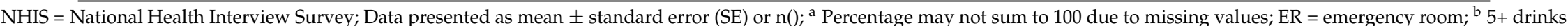
on at least 2 days among men and women in 2013 and $4+$ drinks; on at least 2 days among women in 2014 only; ${ }^{c}$ heavy drinking $=>2$ drinks per day for men and $>1$ drink per day for women; ${ }^{\mathrm{d}}$ Overweight $=\geq 25 \mathrm{~kg} / \mathrm{m}^{2} ;$ e Obesity $=\geq 25 \mathrm{~kg} / \mathrm{m}^{2} ; \mathrm{HIV}=$ Human Immunodeficiency Virus; Note. All estimates are weighted for the survey's complex sampling design. 


\subsection{Sociodemographic and Sleep Characteristics by Race and Sex Living in a Mobile Home/Trailer}

Among participants who live in a mobile home/trailer, black men were more likely than their white counterparts to have a less than high school education (34\% versus $24 \%$ ), an annual household income $<\$ 35,000$ (68\% versus $57 \%$ ), an occupational status of laborer $(84 \%$ versus $75 \%)$, a residence in the South ( $95 \%$ versus $55 \%$ ), and a fair/poor self-reported health status ( $35 \%$ versus $27 \%$ ). White men living in mobile homes/trailers were more likely to be short sleepers ( $34 \%$ versus $29 \%$ ) but less likely ( $12 \%$ versus $19 \%$ ) to be long sleepers compared to black men. Black men were generally as likely to report the recommended amount of sleep ( $52 \%$ versus $54 \%$ ) as white men.

Black women were more likely than white women to have a less than high school education ( $29 \%$ versus $23 \%$ ), have an annual household income less than $\$ 35,000(74 \%$ versus $62 \%)$, have an occupational status of laborer ( $54 \%$ versus $38 \%$ ), a residence in the South ( $96 \%$ versus $57 \%$ ), and health status reported as fair/poor ( $34 \%$ versus $28 \%$ ) than white women. While black women were equally likely to report the recommended amount of sleep (53\% versus $51 \%$ ) as white women, white women living in mobile homes/trailers were more likely to be short sleepers ( $37 \%$ versus $31 \%$ ) but less likely to be long sleepers ( $12 \%$ versus $16 \%$ for women) compared to their black counterparts.

\subsection{Prevalence Ratios of Sleep by Housing Type in the Overall Population}

Fully adjusted prevalence ratios for sleep duration and difficulties by housing type are shown in Table 2. The adjusted prevalence of short sleep was higher for men (PR $=1.05$ (95\% confidence interval (CI): 1.02-1.08)) and women ( $\mathrm{PR}=1.07$ (95\% CI: 1.04-1.09)) in mobile homes/trailers compared to those living in houses/apartments. Long sleep duration was more prevalent among men (PR $=1.09$ (95\% CI: 1.03-1.15)) but not women ( $\mathrm{PR}=1.03$ (95\% CI: 0.98-1.08)) in mobile homes/trailers compared to their counterparts in houses/apartments. In models additionally adjusted for obesity, type 2 diabetes, and hypertension, the effect estimates were similar, and thus the results did not appreciably change (Supplementary Materials Table S1). There were no differences in reported sleep difficulties between men and women in mobile homes/trailers compared to those in homes/apartments.

Table 2. Fully Adjusted Prevalence Ratios for Sleep Duration and Sleep Difficulties for Mobile Home/Trailer Residents Compared to People in Houses/Apartments, among U.S. Men and Women (Black and White): National Health Interview Survey, 2004-2015 ( $N=226,208)$.

\begin{tabular}{|c|c|c|c|c|}
\hline & House/Apartment & Mobile Home/Trailer & House/Apartment & Mobile Home/Trailer \\
\hline & \multicolumn{2}{|c|}{ Men: Overall $(N=102,108)$} & \multicolumn{2}{|c|}{ Women: Overall $(N=124,100)$} \\
\hline \multicolumn{5}{|l|}{ Sleep duration } \\
\hline$<7$ versus $7-8 \mathrm{~h}$ & 1.0 & $1.05(1.02-1.08)$ & 1.0 & $1.07(1.04-1.09)$ \\
\hline$\geq 9$ versus $7-8 \mathrm{~h}$ & 1.0 & $1.09(1.03-1.15)$ & 1.0 & $1.03(0.98-1.08)$ \\
\hline Trouble falling asleep (yes) * & 1.0 & $1.01(0.97-1.06)$ & 1.0 & $1.02(0.98-1.05)$ \\
\hline Trouble staying asleep (yes) * & 1.0 & $1.01(0.97-1.06)$ & 1.0 & $1.01(0.98-1.05)$ \\
\hline Days woke up feeling rested (most) * & 1.0 & $0.98(0.95-1.02)$ & 1.0 & $0.92(0.89-0.96)$ \\
\hline Times took sleep medication last week $\geq 1$ ) * & 1.0 & $1.00(0.93-1.08)$ & 1.0 & $1.01(0.95-1.07)$ \\
\hline
\end{tabular}

PR = Prevalence Ratio; CI = Confidence Interval; Models are adjusted for age, educational attainment, income, occupational class, health status, and region of residence. All estimates are weighted for the survey's complex sampling design. Boldface indicates statistically significant results at the 0.05 level. * Data available from 2013-2015.

\subsection{Prevalence Ratios of Sleep by Race and Housing Type}

The adjusted prevalence ratios for racial differences in sleep duration and difficulties by housing type for men and women are displayed in Table 3. Compared to white men who live in a house/apartment, the adjusted prevalence was $26 \%(\mathrm{PR}=1.26$ (95\% CI: 1.21-1.30)) higher among black men who reported $<7 \mathrm{~h}$ of sleep and $17 \%$ higher among those who reported $>8 \mathrm{~h}$ of sleep $(\mathrm{PR}=1.17$ (95\% CI: 1.09-1.27)). Also, black men who lived in a mobile home/trailer had a 36\% (PR $=1.36$ (95\% CI: 1.11-1.67)) higher prevalence of sleeping $>8 \mathrm{~h}$ relative to white men who lived in a mobile home/trailer. There were no differences observed between white and black men who live in a mobile home/trailer as it relates to reporting $<7 \mathrm{~h}$ of sleep. 
Table 3. Fully Adjusted Prevalence Ratios for Sleep Duration and Sleep Difficulties Compared to Housing Type among U.S. Black Men (referent White Men) and Black Women (referent White Women), National Health Interview Survey, 2004-2015 $(N=226,208)$.

\begin{tabular}{|c|c|c|c|c|}
\hline & \multicolumn{2}{|c|}{ Men } & \multicolumn{2}{|c|}{ Women } \\
\hline & House/Apartment & Mobile Home/Trailer & House/Apartment & Mobile Home/Trailer \\
\hline & Black: 15,410 & Black: 758 & Black: 23,795 & Black: 939 \\
\hline Sample size & White: 80,857 & White: 5083 & White: 93,812 & White: 5554 \\
\hline \multicolumn{5}{|l|}{ Sleep duration } \\
\hline$<7$ versus $7-8 \mathrm{~h}$ & $1.26(1.21-1.30)$ & $0.90(0.77-1.06)$ & $1.24(1.20-1.27)$ & $0.89(0.74-1.06)$ \\
\hline$\geq 9$ versus $7-8 \mathrm{~h}$ & $1.17(1.09-1.27)$ & $1.36(1.11-1.67)$ & $1.06(1.00-1.13)$ & $1.19(0.93-1.53)$ \\
\hline Trouble falling asleep (yes) * & $0.84(0.78-0.90)$ & $0.83(0.56-1.23)$ & $0.84(0.80-0.89)$ & $0.77(0.64-0.94)$ \\
\hline Trouble staying asleep (yes) * & $0.85(0.80-0.91)$ & $0.71(0.51-0.99)$ & $0.82(0.78-0.86)$ & $0.73(0.56-0.95)$ \\
\hline Days woke up feeling rested (most)* & $1.05(1.01-1.09)$ & $1.10(0.92-1.32)$ & $1.06(1.02-1.10)$ & $1.59(1.30-1.94)$ \\
\hline Times took sleep medication last week $\geq 1$ ) & $0.71(0.61-0.82)$ & $0.57(0.30-1.09)$ & $0.67(0.61-0.73)$ & $0.33(0.22-0.50)$ \\
\hline
\end{tabular}

PR = Prevalence Ratio; CI = Confidence Interval; Adjusted for age, educational attainment, income, occupational class, health status, and region of residence. Note. All estimates are weighted for the survey's complex sampling design. Boldface indicates statistically significant results at the 0.05 level. * data available from $2013-2015$

Black women who lived in a house/apartment had a 24\% (PR = 1.24 (95\% CI: 1.20-1.27)) higher prevalence of reporting sleep $<7 \mathrm{~h}$ relative to white women who lived in a house/apartment. There was no difference in reporting $>8 \mathrm{~h}$ of sleep between black and white women who live in a house/apartment. Similarly, the adjusted prevalence of reporting $<7 \mathrm{~h}$ of sleep ( $\mathrm{PR}=0.89$ (95\% CI: $0.74-1.06)$ ) or $>8 \mathrm{~h}$ of sleep (PR $=1.19$ (95\% CI: 0.93-1.53)) was not different between black and white women who lived in a mobile home/trailer.

Regarding sleep difficulties, compared to white men who lived in a house/apartment, black men had a lower adjusted prevalence of having trouble falling asleep ( $\mathrm{PR}=0.84(95 \% \mathrm{CI}$ : $0.78-0.90)$ ) and having trouble staying asleep ( $\mathrm{PR}=0.85$ (95\% CI: $0.80-0.91)$ ), but had a higher adjusted prevalence of reporting waking up feeling rested on most days (PR $=1.05$ (95\% CI: 1.01-1.09)). Black men were also less likely to take a sleep medication at least once in the prior week (PR $=0.71$ (95\% CI: 0.61-0.82)) than white men. Among men who lived in a mobile home/trailer, black men had a lower adjusted prevalence of having trouble staying asleep ( $\mathrm{PR}=0.71(95 \% \mathrm{CI}$ : $0.51-0.99)$ ) than white men, but there were no statistically significant differences in the adjusted prevalence of having trouble falling asleep, waking up feeling rested on most days, or reporting frequency of taking a sleep medication in the last week one or more times.

Compared to white women who resided in a house/apartment, their black counterparts reported a significantly lower adjusted prevalence of having trouble falling asleep (PR $=0.84$ (95\% CI: $0.80-0.89)$ ) and having trouble staying asleep ( $\mathrm{PR}=0.82(95 \% \mathrm{CI}$ : $0.78-0.86)$ ), but had a higher adjusted prevalence of reporting waking up feeling rested on most days (PR = 1.06 (95\% CI: 1.02-1.10)). Black women were also less likely to take a sleep medication in the prior week ( $\mathrm{PR}=0.67$ (95\% CI: $0.61-0.73$ ) ) compared to white women in a house/apartment. A comparable pattern was observed for women who lived in a mobile home/trailer. For example, black women had a lower adjusted prevalence of having trouble falling asleep (PR $=0.77$ (95\% CI: 0.64-0.94)) and having trouble staying asleep (PR $=0.73$ (95\% CI: $0.56-0.95)$ ), but had a $59 \%$ (PR = 1.59 (95\% CI: 1.30-1.94)) higher prevalence of reporting waking up feeling rested most of the days compared to white women who also lived in a mobile home/trailer. Black women compared to white women in mobile homes/trailers were also less likely to take sleep medications in the prior week ( $\mathrm{PR}=0.33$ (95\% CI: 0.22-0.50)).

In secondary analyses, additional adjustment for obesity, type 2 diabetes, and hypertension resulted in similar effect estimates, which did not appreciably change the results (Supplementary Materials Table S2).

\section{Discussion}

In a nationally representative sample of black and white U.S. adults, we found important disparities in sleep duration and sleep difficulties by housing type overall as well as by race within housing type among both men and women. Our results support the idea that residing in a home or apartment is generally better for sleep duration than residing in mobile homes or trailers. 
For instance, individuals who lived in mobile homes/trailers were more likely to have shorter sleep duration and report fair/poor health in comparison to those who lived in a house or apartment. Before adjustments, short sleep duration was more prevalent among blacks compared to whites living in a house/apartment and more prevalent among whites compared to blacks living in mobile homes/trailers. After adjustments, there were striking racial disparities in sleep duration among those who lived a house or apartment that remained. Black men and women who resided in a house or apartment were more likely to report a habitual short sleep duration and were more likely to report their health as fair or poor compared to their white counterparts. However, no significant racial difference was observed for those in mobile homes/trailers with the exception of black men, who were significantly more likely than white men to report long sleep duration. Black men and women who lived in homes or apartments had a lower prevalence of sleep difficulties and were more likely to report waking feeling rested relative to their white counterparts. These black/white differences in sleep difficulties did not apply to men in trailers/mobile homes as there was no significant difference except among black men who were less likely to report trouble staying asleep. Black women in a mobile home/trailer had a lower prevalence of sleep difficulties and a higher prevalence of feeling rested most days compared to their white counterparts. Given the racial disparities in housing type, it is important to deeply understand these findings as a potential effective approach to improve sleep health and address health disparities.

Although the scientific evidence describing the impact of housing conditions on sleep is sparse, individuals who reside in mobile homes/trailers likely have greater exposure to adverse housing and neighborhood conditions that can negatively impact health compared to individuals who reside in houses/apartments. Mobile homes tend to be concentrated in more economically deprived neighborhoods, and prior studies have shown that deprived neighborhoods with crime, violence, or disorder are associated with shorter sleep duration [3,23]. More studies need to also assess the impact on sleep quality. While the mechanisms underlying the association are unclear, mobile homes/trailers may represent a particularly vulnerable/adverse environment where residents are more likely to experience stressors (e.g., housing and other financial-related insecurities; safety concerns) that impact sleep compared to those in more relatively stable environments, such as houses or apartments. These individuals may also be more likely to have overt or even preclinical health conditions that affect or are affected by suboptimal sleep.

Both black men and women reported shorter sleep duration relative to white men and women living in houses and apartments. The observed racial disparity in sleep duration may be attributable to various factors. For instance, black residents may experience more perceived stress, which is associated with short sleep duration [23]. In the current study sample, black compared to white participants were less likely to be homeowners, and thus may represent either a more transient or lower SES population, which is also independently associated with short sleep duration [34]. There was no racial difference in short sleep duration among those in mobile homes/trailers. Blacks who reside in homes or apartments tend to be of higher SES than those living in mobile homes, and a study by Jackson et al. found that blacks of higher SES appear more likely to be short sleepers than their lower SES counterparts [20]. Moreover, data suggest that highly educated blacks may be more vulnerable to the effects of stress on sleep duration than blacks with a lower level of educational attainment [34]. There are additional data suggesting that health behaviors (e.g., alcohol consumption) and chronic conditions (e.g., obesity, hypertension) are similar when blacks and whites live in similar environments [35-38]. Furthermore, disparities in wealth as well as access to positive material and intangible resources are likely to be much wider among blacks and whites who live in houses and even apartments than in mobile homes as the quality of the housing structures and surrounding amenities likely differ greatly by SES and race. Regarding individuals in houses/apartments, the percentage reporting low income was almost double for black versus white men, and black versus white women; this black-white gap in low-income was reduced in mobile homes/trailers, although poverty was worse. Thus, we expected to observe that the racial disparity in sleep would be lessened in mobile homes/trailers. 
In both homes/apartments and mobile homes/trailers, black men were significantly more likely than white men to report long sleep duration. Prior studies using data from nationally representative samples have also demonstrated that black adults have a higher prevalence of both short and long sleep durations, which may indicate more variation in habitual sleep time [39]. This finding has significant implications regarding health outcomes. There are links between long sleep duration and increased mortality and incident cardiovascular disease [40]. Although the levels of clinical significance have not been established regarding sleep duration, the observed prevalence estimates of short and long sleep duration comparing black and white adults were similar to the effect sizes for well-known associations, such as short sleep duration and various cardiovascular outcomes (diabetes (odds ratio $(\mathrm{OR})=1.37$, $95 \%$ CI 1.22-1.53), hypertension $(1.17,1.09-1.26)$, obesity $(1.38,1.25-1.53))$ [41]. Future research should explore features of the housing environment that may promote suboptimal sleep durations among black man, particularly in efforts to reduce subsequent adverse health outcomes.

Regarding racial differences in reports of sleep difficulties, blacks have been shown to be less likely to self-report sleep complaints [42], but their sleep is consistently worse than whites based on objective sleep measures [43]. Future studies should investigate whether the observed finding that black men and women report less sleep difficulties is an accurate finding or due to misclassification. If due to misclassification, objective measures of sleep quality will be necessary in future studies. Further, disparities in sleep difficulties may be patterned differently among lower SES blacks and whites. Lower SES whites who reside in mobile homes may be more vulnerable to the effects of poor housing on sleep because whites are less likely to have experienced historical disadvantage related to concentrated poverty [44]. Studies have demonstrated that white men and women relative to their black counterparts tend to be less resilient to adversities due to the infrequent experiences of stressors, which have been shown to have a stronger impact on physical and mental health [44-46]. In the current study, more white men and women reported feeling depressed than their black counterparts in mobile homes, which supports the notion that white men and women may be more (or more likely to report being) mentally affected by their disadvantaged circumstance. Black individuals are a historically disadvantaged population across multiple generations, and research has shown that individuals can become desensitized and adopt behavioral techniques (e.g., alcohol and other substance abuse) in an attempt to buffer the negative mental health consequences with cumulative exposures to multiple stressors [47], which may help to explain this finding. Nonetheless, future studies are needed to better understand these relationships.

Our study has several limitations. This was a cross-sectional analysis, which precludes inferences related to causation because of difficulty in establishing temporality, avoiding reverse causation and potential endogeneity bias, and measuring the dynamic nature of neighborhoods as well as more immediate housing conditions (e.g., poor/substandard quality structures, overcrowding, unstable neighborhood). Furthermore, our sleep duration measure was based on self-report, which is prone to measurement error as individuals generally tend to overestimate their sleep duration [48]. Due to the NHIS data collection methods, we were also unable to differentiate between living in a house versus an apartment. These dwellings likely have differential social and environmental exposures that impact health. Additionally, there were fewer participants who resided in a mobile home/trailer compared to a house/apartment; however, the sample size was sufficient to provide robust estimates for our main research question. Furthermore, there are important potential confounders, such as psychosocial stress and urban density, that were unmeasured and several important confounders (e.g., income) were adjusted for in a crude manner, which could result in residual confounding. We were also unable to assess housing insecurity or level of mobility, which likely varies by housing type. We also could not assess endogeneity and did not include institutionalized (e.g., nursing homes) populations.

Despite the limitations, this study has important strengths. For instance, these data are based on a nationally representative large sample of black and white men and women, which allowed for robust estimation despite stratification. We also provided new data regarding racial differences in sleep duration among those who reside in apartments/houses, which helps to identify vulnerable 
populations that need tailored interventions focused on reducing sleep disparities. Furthermore, we included directly estimated prevalence ratios rather than the more difficult to interpret odds ratios, which simply approximate prevalence ratios. Lastly, the analysis included a large number of serial sleep measurements across survey years from 2004 to 2015.

\section{Conclusions}

In conclusion, these findings demonstrate that individuals in mobile/trailer homes, which could serve as a marker of suboptimal housing, may have poorer sleep than those in houses/apartments. Furthermore, there were also important within and between race-sex group differences in sleep duration and difficulties by housing type that need to be further investigated. The physical and social features of housing- and neighborhood-level factors that promote poor sleep need to be illuminated to eventually develop tailored intervention strategies at multiple levels that improve sleep in the population while addressing disparities.

Supplementary Materials: The following are available online at http:/ /www.mdpi.com/1660-4601/15/4/564/s1, Table S1: Fully Adjusted Prevalence Ratios for Sleep Duration and Quality Measures for Mobile Home/Trailer Dwellers Compared to People in Houses/Apartments, among U.S. Men and Women (Black and White): National Health Interview Survey, 2004-2015 $(N=226,208)$, Table S2: Fully Adjusted Prevalence Ratios for Sleep Duration and Sleep Quality Indicators in Relation to Housing Type among U.S. Black Men (referent White Men) and Black Women (referent White Women), National Health Interview Survey, 2004-2015 $(N=226,208)$.

Acknowledgments: This work was funded by the Intramural Program at the NIH, National Institute of Environmental Health Sciences (Z1AES103325-01) and by the extramural program at the National Institute of Heart Lung and Blood (K01HL138211) and the National Institute on Minority Health and Health Disparities (U54MD000214). These data were presented, in part, at the Society for Epidemiologic Research Annual Meeting in Seattle, Washington in 20-23 June 2017.

Author Contributions: Authors: Dayna A. Johnson, Roland J. Thorpe, Jr., W. Braxton Jackson, John A. McGrath, Chandra L. Jackson. Study concept: C.L. Jackson. Study design: D.A. Johnson, R.J. Thorpe, W.B. Jackson, J.A. McGrath, C.L. Jackson. Acquisition of data: W.B. Jackson, C.L. Jackson. Statistical analysis: W.B. Jackson, C.L. Jackson. Interpretation of data: D.A. Johnson, R.J. Thorpe, W.B. Jackson, J.A. McGrath, C.L. Jackson. Drafting of the manuscript: D.A. Johnson, R.J. Thorpe, C.L. Jackson. Critical revision of the manuscript for important intellectual content: D.A. Johnson, R.J. Thorpe, W.B. Jackson, J.A. McGrath, C.L. Jackson. Administrative, technical, and material support: C.L. Jackson. Obtaining funding and study supervision: C.L. Jackson. Final approval: D.A. Johnson, R.J. Thorpe, W.B. Jackson, J.A. McGrath, C.L. Jackson.

Conflicts of Interest: The authors declare no conflict of interest.

\section{References}

1. Shaw, M. Housing and public health. Annu. Rev. Public Health 2004, 25, 397-418. [CrossRef] [PubMed]

2. Krieger, J.; Higgins, D.L. Housing and health: Time again for public health action. Am. J. Public Health 2002, 92, 758-768. [CrossRef] [PubMed]

3. Johnson, D.A.; Simonelli, G.; Moore, K.; Billings, M.; Mujahid, M.S.; Rueschman, M.; Kawachi, I.; Redline, S.; Diez Roux, A.V.; Patel, S.R. The Neighborhood Social Environment and Objective Measures of Sleep in the Multi-Ethnic Study of Atherosclerosis. Sleep 2017, 40. [CrossRef] [PubMed]

4. Johnson, D.A.; Brown, D.L.; Morgenstern, L.B.; Meurer, W.J.; Lisabeth, L.D. The association of neighborhood characteristics with sleep duration and daytime sleepiness. Sleep Health 2015, 1, 148-155. [CrossRef] [PubMed]

5. Hume, K.I.; Brink, M.; Basner, M. Effects of environmental noise on sleep. Noise Health 2012, 14, $297-302$. [CrossRef] [PubMed]

6. Zanobetti, A.; Redline, S.; Schwartz, J.; Rosen, D.; Patel, S.; O'Connor, G.T.; Lebowitz, M.; Coull, B.A.; Gold, D.R. Associations of $\mathrm{PM}_{10}$ with sleep and sleep-disordered breathing in adults from seven U.S. urban areas. Am. J. Respir. Crit. Care Med. 2010, 182, 819-825. [CrossRef] [PubMed]

7. Schootman, M.; Andresen, E.M.; Wolinsky, F.D.; Malmstrom, T.K.; Miller, J.P.; Yan, Y.; Miller, D.K. The effect of adverse housing and neighborhood conditions on the development of diabetes mellitus among middle-aged African Americans. Am. J. Epidemiol. 2007, 166, 379-387. [CrossRef] [PubMed] 
8. Morello-Frosch, R.; Zuk, M.; Jerrett, M.; Shamasunder, B.; Kyle, A.D. Understanding the cumulative impacts of inequalities in environmental health: Implications for policy. Health Aff. 2011, 30, 879-887. [CrossRef] [PubMed]

9. Mullington, J.M.; Haack, M.; Toth, M.; Serrador, J.M.; Meier-Ewert, H.K. Cardiovascular, inflammatory, and metabolic consequences of sleep deprivation. Prog. Cardiovasc. Dis. 2009, 51, 294-302. [CrossRef] [PubMed]

10. Chambers, E.C.; Pichardo, M.S.; Rosenbaum, E. Sleep and the Housing and Neighborhood Environment of Urban Latino Adults Living in Low-Income Housing: The AHOME Study. Behav. Sleep Med. 2016, 14, 169-184. [CrossRef] [PubMed]

11. Simonelli, G.; Leanza, Y.; Boilard, A.; Hyland, M.; Augustinavicius, J.L.; Cardinali, D.P.; Vallières, A.; Pérez-Chada, D.; Vigo, D.E. Sleep and quality of life in urban poverty: The effect of a slum housing upgrading program. Sleep 2013, 36, 1669-1676. [CrossRef] [PubMed]

12. Solari, C.D.; Mare, R.D. Housing crowding effects on children's wellbeing. Soc. Sci. Res. 2012, 41, $464-476$. [CrossRef] [PubMed]

13. Griefahn, B.; Marks, A.; Robens, S. Noise emitted from road, rail and air traffic and their effects on sleep. J. Sound Vib. 2006, 295, 129-140. [CrossRef]

14. Jakovljevic, B.; Belojevic, G.; Paunovic, K.; Stojanov, V. Road traffic noise and sleep disturbances in an urban population: Cross-sectional study. Croat. Med. J. 2006, 47, 125-133. [PubMed]

15. Johnson, D.A.; Drake, C.; Joseph, C.L.; Krajenta, R.; Hudgel, D.W.; Cassidy-Bushrow, A.E. Influence of neighbourhood-level crowding on sleep-disordered breathing severity: Mediation by body size. J. Sleep Res. 2015, 24, 559-565. [CrossRef] [PubMed]

16. Liu, X.; Liu, L.; Wang, R. Bed sharing, sleep habits, and sleep problems among Chinese school-aged children. Sleep 2003, 26, 839-844. [CrossRef] [PubMed]

17. Sandberg, J.C.; Talton, J.W.; Quandt, S.A.; Chen, H.; Weir, M.; Doumani, W.R.; Chatterjee, A.B.; Arcury, T.A. Association between housing quality and individual health characteristics on sleep quality among Latino farmworkers. J. Immigr. Minor. Health 2014, 16, 265-272. [CrossRef] [PubMed]

18. Chung, S.; Wilson, K.E.; Miller, A.L.; Johnson, D.A.; Lumeng, J.C.; Chervin, R.D. Home Sleeping Conditions and Sleep Quality in Low-Income Preschool Children. Sleep Med. Res. 2014, 5, 29-32. [CrossRef]

19. Chen, X.; Wang, R.; Zee, P.; Lutsey, P.L.; Javaheri, S.; Alcántara, C.; Jackson, C.L.; Williams, M.A.; Redline, S. Racial/Ethnic Differences in Sleep Disturbances: The Multi-Ethnic Study of Atherosclerosis (MESA). Sleep 2015, 38, 877-888. [CrossRef] [PubMed]

20. Jackson, C.L.; Redline, S.; Kawachi, I.; Williams, M.A.; Hu, F.B. Racial disparities in short sleep duration by occupation and industry. Am. J. Epidemiol. 2013, 178, 1442-1451. [CrossRef] [PubMed]

21. Hale, L.; Do, D.P. Racial differences in self-reports of sleep duration in a population-based study. Sleep 2007, 30, 1096-1103. [CrossRef] [PubMed]

22. Bureau, U.S.C. American Housing Survey for the United States: 2011; Current Housing Reports, Series H150/11; U.S. Government Printing Office: Washington, DC, USA, 2013.

23. Johnson, D.A.; Lisabeth, L.; Hickson, D.; Johnson-Lawrence, V.; Samdarshi, T.; Taylor, H.; Diez Roux, A.V. The Social Patterning of Sleep in African Americans: Associations of Socioeconomic Position and Neighborhood Characteristics with Sleep in the Jackson Heart Study. Sleep 2016, 39, 1749-1759. [CrossRef] [PubMed]

24. Desantis, A.S.; Diez Roux, A.V.; Moore, K.; Baron, K.G.; Mujahid, M.S.; Nieto, F.J. Associations of neighborhood characteristics with sleep timing and quality: The Multi-Ethnic Study of Atherosclerosis. Sleep 2013, 36, 1543-1551. [CrossRef] [PubMed]

25. Jackson, C.L.; Redline, S.; Emmons, K.M. Sleep as a potential fundamental contributor to disparities in cardiovascular health. Annu. Rev. Public Health 2015, 36, 417-440. [CrossRef] [PubMed]

26. Cappuccio, F.P.; Cooper, D.; D’Elia, L.; Strazzullo, P.; Miller, M.A. Sleep duration predicts cardiovascular outcomes: A systematic review and meta-analysis of prospective studies. Eur. Heart J. 2011, 32, 1484-1492. [CrossRef] [PubMed]

27. National Center for Health Statistics CfDCaPNHISH, MD. Available online: http:/ /www.cdc.gov/nchs / nhis.htm (accessed on 19 March 2018).

28. Jackson, C.L.; Hu, F.B.; Redline, S.; Williams, D.R.; Mattei, J.; Kawachi, I. Racial/ethnic disparities in short sleep duration by occupation: The contribution of immigrant status. Soc. Sci. Med. 2014, 118, 71-79. [CrossRef] [PubMed] 
29. Consensus Conference Panel; Watson, N.F.; Badr, M.S.; Belenky, G.; Bliwise, D.L.; Buxton, O.M.; Buysse, D.; Dinges, D.F.; Gangwisch, J.; Grandner, M.A.; et al. Recommended Amount of Sleep for a Healthy Adult: A Joint Consensus Statement of the American Academy of Sleep Medicine and Sleep Research Society. J. Clin. Sleep Med. 2015, 11, 591-592. [PubMed]

30. Jackson, C.L. Determinants of racial/ethnic disparities in disordered sleep and obesity. Sleep Health 2017, 3, 401-415. [CrossRef] [PubMed]

31. World Health Organization Expert Committee on Physical Status. Use and Interpretation of Anthropometry. Physical Status: The Use and Interpretation of Anthropometry: Report of a WHO Expert Committee; WHO: Geneva, Switzerland, 1995.

32. Wolters, K.M. Introduction to Variance Estimation; Springer: New York, NY, USA, 1990.

33. Barros, A.J.; Hirakata, V.N. Alternatives for logistic regression in cross-sectional studies: An empirical comparison of models that directly estimate the prevalence ratio. BMC Med. Res. Methodol. 2003, 3, 21. [CrossRef] [PubMed]

34. Johnson, D.A.; Lisabeth, L.; Lewis, T.T.; Sims, M.; Hickson, D.A.; Samdarshi, T.; Taylor, H.; Diez Roux, A.V. The Contribution of Psychosocial Stressors to Sleep among African Americans in the Jackson Heart Study. Sleep 2016, 39, 1411-1419. [CrossRef] [PubMed]

35. Thorpe, R.J., Jr.; Kennedy-Hendricks, A.; Griffith, D.M.; Bruce, M.A.; Coa, K.; Bell, C.N.; Young, J.; Bowie, J.V.; LaVeist, T.A. Race, Social and Environmental Conditions, and Health Behaviors in Men. Fam. Community Health 2015, 38, 297-306. [CrossRef] [PubMed]

36. Fesahazion, R.G.; Thorpe, R.J., Jr.; Bell, C.N.; LaVeist, T.A. Disparities in alcohol use: Does race matter as much as place? Prev. Med. 2012, 55, 482-484. [CrossRef] [PubMed]

37. Bleich, S.N.; Thorpe, R.J., Jr.; Sharif-Harris, H.; Fesahazion, R.; Laveist, T.A. Social context explains race disparities in obesity among women. J. Epidemiol. Community Health 2010, 64, 465-469. [CrossRef] [PubMed]

38. LaVeist, T.; Pollack, K.; Thorpe, R., Jr.; Fesahazion, R.; Gaskin, D. Place, not race: Disparities dissipate in southwest Baltimore when blacks and whites live under similar conditions. Health Aff. 2011, 30, 1880-1887. [CrossRef] [PubMed]

39. Nunes, J.; Jean-Louis, G.; Zizi, F.; Casimir, G.J.; von Gizycki, H.; Brown, C.D.; McFarlane, S.I. Sleep duration among black and white Americans: Results of the National Health Interview Survey. J. Natl. Med. Assoc. 2008, 100, 317-322. [CrossRef]

40. Jike, M.; Itani, O.; Watanabe, N.; Buysse, D.J.; Kaneita, Y. Long sleep duration and health outcomes: A systematic review, meta-analysis and meta-regression. Sleep Med. Rev. 2017. [CrossRef] [PubMed]

41. Itani, O.; Jike, M.; Watanabe, N.; Kaneita, Y. Short sleep duration and health outcomes: A systematic review, meta-analysis, and meta-regression. Sleep Med. 2017, 32, 246-256. [CrossRef] [PubMed]

42. Grandner, M.A.; Patel, N.P.; Gehrman, P.R.; Xie, D.; Sha, D.; Weaver, T.; Gooneratne, N. Who gets the best sleep? Ethnic and socioeconomic factors related to sleep complaints. Sleep Med. 2010, 11, 470-478. [CrossRef] [PubMed]

43. Lauderdale, D.S.; Knutson, K.L.; Yan, L.L.; Rathouz, P.J.; Hulley, S.B.; Sidney, S.; Liu, K. Objectively measured sleep characteristics among early-middle-aged adults: The CARDIA study. Am. J. Epidemiol. 2006, 164, 5-16. [CrossRef] [PubMed]

44. Assari, S.; Lankarani, M.M. Association between Stressful Life Events and Depression; Intersection of Race and Gender. J. Racial Ethn. Health Dispar. 2016, 3, 349-356. [CrossRef] [PubMed]

45. Assari, S.; Lankarani, M.M. Depressive Symptoms Are Associated with More Hopelessness among White than Black Older Adults. Front. Public Health 2016, 4, 82. [CrossRef] [PubMed]

46. Assari, S. Race, sense of control over life, and short-term risk of mortality among older adults in the United States. Arch. Med. Sci. 2017, 13, 1233-1240. [CrossRef] [PubMed]

47. Jackson, J.S.; Knight, K.M.; Rafferty, J.A. Race and unhealthy behaviors: Chronic stress, the HPA axis, and physical and mental health disparities over the life course. Am. J. Public Health 2010, 100, 933-939. [CrossRef] [PubMed]

48. Lauderdale, D.S.; Knutson, K.L.; Yan, L.L.; Liu, K.; Rathouz, P.J. Self-reported and measured sleep duration: How similar are they? Epidemiology 2008, 19, 838-845. [CrossRef] [PubMed]

(C) 2018 by the authors. Licensee MDPI, Basel, Switzerland. This article is an open access article distributed under the terms and conditions of the Creative Commons Attribution (CC BY) license (http://creativecommons.org/licenses/by/4.0/). 